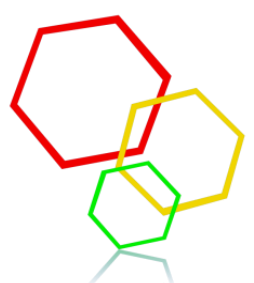

\title{
Journal of Chemistry and Technologies
}

pISSN 2663-2934 (Print), ISSN 2663-2942 (Online).

journal homepage: http://chemistry.dnu.dp.ua

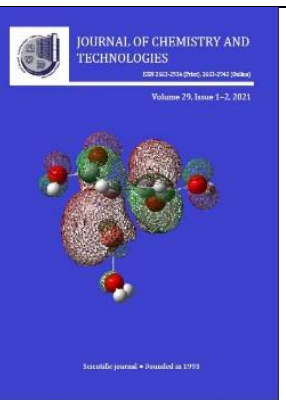

UDC 543.421:637.07

ANALYSIS OF WATER AND BOTTOM SEDIMENTS OF THE TIGER RIVER (IRAQ) USING ULTRASONIC TREATMENT, NONIONIC SURFACE ACTIVE SUBSTANCES AND $\beta$ DIKETONATES OF METALS AS STANDARD SAMPLES

Oleg I. Yurchenko, Tetyana V. Chernozhuk, Alexandr N. Baklanov*, Oleksii A. Kravchenko

V.N. Karazin Kharkiv National University, 4 Svobody Sq., 61022 Kharkiv, Ukraine Received April 2021; accepted May 2021; available online 20 July 2021

\begin{abstract}
Methods of atomic absorption spectroscopy and atomic emission spectroscopy with inductively coupled plasma were used to determine the content of copper, cadmium, zinc and lead in the water of the Tigris River (Iraq). It is shown that the results of both methods are equally accurate, the difference between them is insignificant and is explained by a random error. The use of Triton X-100 made it possible to reduce the detection limits of metals by 1.5-5 times compared to the standard approach. The mercury content in bottom sediments was determined by the $X$-ray fluorescence method and the atomic absorption method of cold steam. The found concentration of mercury in the bottom sediments of the Tigris River exceeded, on average, 1.5-2 times the standard values established for it in Iraq $(0.2 \mathrm{mg} / \mathrm{kg})$. New reference materials of composition based on acetylacetonates of copper, zinc, cadmium, lead and mercury (I) dimedonate, metal $\beta$-diketonates made it possible to increase the precision and accuracy of measurements due to the similarity of the chemical composition of the analyzed and reference samples.

Key words: copper; zinc; lead; cadmium; mercury; atomic absorption spectroscopy; atomic emission spectroscopy with inductively coupled plasma; X-ray fluorescence analysis; Triton X-100; $\beta$-diketonates of metals.

\section{АНАЛІЗ ВОДИ І ДОННИХ ВІДКЛАДНЕНЬ РІЧКИ ТИГР (ІРАК) 3 ВИКОРИСТАННЯМ УЛЬТРАЗВУКОВОЇ ОБРОБКИ, НЕІОННИХ ПОВЕРХНЕВО-АКТИВНИХ РЕЧОВИН І $\beta-$ ДИКЕТОНАТІВ МЕТАЛІВ ЯК СТАНДАРТНИХ ЗРАЗКІВ СКЛАДУ \\ Олег І. Юрченко, Тетяна В. Черножук, Олександр М. Бакланов, Олексій А. Кравченко. Харківський національний університет імені В.Н. Каразіна, пл. Свободи, 4, Харків, 61022, Україна}

\begin{abstract}
Антоація
Методами атомно-абсорбційної спектроскопії і атомно-емісійної спектроскопії з індуктивно-зв'язаною плазмою проведено визначення вмісту купруму, кадмію, цинку і плюмбуму у воді річки Тигр (Ірак). Показано, що результати обох методів є рівноточними, розбіжність між ними є незначимою і виправданою випадковими похибками. Використання Тритон X-100 дозволило знизити межі виявлення металів в 1,5-5 разів у порівнянні зі стандартним підходом. Рентгенофлуоресцентним методом і атомно-абсорбційним методом холодного пару визначено вміст меркурію в донних відкладеннях. Знайдена концентрація меркурію в донних відкладеннях річки Тигр перевищувала в середньому в 1.5-2 рази нормативні показники, встановлені для неї в Іраку (0.2 мг / кг). Нові стандартні зразки складу на основі ацетилацетонатів купруму, цинку, кадмію, плюмбуму і дімедонату меркурію (I), $\beta$-дикетонатів металів дозволили підвищити прецизійність і точність вимірювань за рахунок зближення хімічного складу аналізованих зразків і зразків порівняння.

Ключові слова: купрум; цинк; плюмбум; кадмій; меркурій; атомно-абсорбційна спектроскопія; атомно-емісійна спектроскопія з індуктивно зв'язаною плазмою; рентгенофлуоресцентний аналіз; тритон X-100; $\beta$-дикетонати металів.
\end{abstract}




\title{
АНАЛИЗ ВОДЫ И ДОННЫХ ОТЛОЖЕНИЙ РЕКИ ТИГР (ИРАК) С ИСПОЛЬЗОВАНИЕМ УЛЬТРАЗВУКОВОЙ ОБРАБОТКИ, НЕИОННЫХ ПОВЕРХНОСТНО-АКТИВНЫХ ВЕЩЕСТВ И $\beta$-ДИКЕТОНАТОВ МЕТАЛЛОВ В КАЧЕСТВЕ СТАНДАРТНЫХ ОБРАЗЦОВ СОСТАВА
}

\author{
Олег И. Юрченко, Татьяна В. Черножук, Александр Н. Бакланов, Алексей А. Кравченко \\ Харьковский национальный университет имени В.Н. Каразина, 61022, Украина Харьков, пл. Свободы, 4
}

\begin{abstract}
Аннотация
Методами атомно-абсорбционной спектроскопии и атомно-эмиссионной спектроскопии с индуктивносвязанной плазмой проведено определение содержания меди, кадмия, цинка и свинца в воде реки Тигр (Ирак). Показано, что результаты обоих методов являются равноточными, расхождение между ними незначимо и оправдано случайным разбросом. Использование Тритон X-100 позволило снизить пределы обнаружения металлов в 1,5-5 раз по сравнению со стандартным подходом. Рентгенофлуоресцентным методом и атомно-абсорбционным методом холодного пара определено содержание ртути в донных отложениях. Найденная концентрация ртути в донных отложениях реки Тигр превышала в среднем в 1.5-2 раза нормативные показатели, установленные для нее в Ираке (0.2 мг/кг). Новые стандартные образцы состава на основании ацетилацетонатов меди, цинка, кадмия, свинца и димедоната ртути (I), $\beta$-дикетонатов металлов позволили повысить прецизионность и точность измерений за счет сближения химического состава анализируемых образцов и образцов сравнения.

Ключевые слова: медь; цинк; свинец; кадмий; ртуть; атомно-абсорбционная спектроскопия; атомно-эмиссионная спектроскопия с индуктивно связанной плазмой; рентгенофлуоресцентный анализ; тритон X-100; $\beta$-дикетонаты металлов.
\end{abstract}

\section{Введение}

K настоящему времени накопились многочисленные данные о вредном воздействии тяжелых металлов на человека, животных и растения [1; 2]. Развитие промышленности, сельского хозяйства, энергетики и транспорта, интенсивная добыча полезных ископаемых привели к поступлению в воздух, воду, почву, растения сотен высокотоксичных химических веществ, одними из которых являются химические элементы, относящиеся к тяжелым металлам [3-9].

Анализ природных и промышленных вод на содержание тяжелых металлов является актуальным в связи с всевозрастающими санитарными требованиями к чистоте разных типов вод. Важным объектом исследования являются донные отложения рек, так как в них накапливаются со временем значительные содержания токсичных веществ $[1 ; 2]$.

Использование воды водоема, в данных отложениях которого находится высокая концентрация тяжелых металлов, может привести к негативным последствиям. Особое значение имеет определение ртути, цинка, меди, свинца, кадмия и т.д. Наиболее широко на современном этапе для определения опасных для окружающей среды и человека металлов применяются пламенный и электротермический варианты атомноабсорбционной спектроскопии, включая спектроскопию холодного пара, атомноэмиссионная спектроскопия с индуктивносвязанной плазмой, рентгенофлуорес- центный анализ [3; 4-6]. Эти методы отвечают требованиям современной «зеленой химии». Продолжается совершенствование и развитие методов пробоподготовки, состав анализируемых растворов модифицируется при помощи поверхностно-активных веществ, а для учета влияния матрицы предлагается использовать специально подобранные стандартные образцы состава [4; 7-18].

Цель работы - разработка усовершенствованных методик атомноабсорбционного, атомно-эмиссионного с индуктивно - связанной плазмой и рентгенофлуоресцентного определения содержание некоторых тяжелых металлов в воде и донных отложениях с использованием ультразвуковой обработки, водных растворов неионных ПАВ и $\beta$-дикетонатов металлов в качестве стандартных образцов состава.

\section{Экспериментальная часть}

Используемые аппаратура, оборудование и реактивы. В работе использовались следующие инструменты. Атомноабсорбционный спектрометр С-115 (пламенный вариант), пламя ацетилен воздух. Атомно-эмиссионный спектрометр с индуктивно-связанной плазмой iCAP 6300 DUO: скорость плазмообразующего потока аргона - 12 л/мин; мощность плазмы - 1350 Вт; скорость дополнительного потока аргона - 1.5 л/мин; режим наблюдения плазмы аксиальный; поток аргона в распылителе 0.55 л/мин; скорость вращения насоса - 50 оборотов/мин; время интегрирования сигнала - 20 с., проводилось 5 параллельных 
Journal of Chemistry and Technologies, 2021, 29(2), 173-178

измерений; аналитическая длина волны, нм: свинец - 220.353; медь - 327.396; цинк 213.856; кадмий $\quad$ - 226.502. Рентгенофлуоресцентный спектрометр VRA30 (регистрация $L_{\alpha}$ линий ртути, которые возбуждалась излучением трубки с молибденовым анодом и напряжением 52 кВ, ток - 75 мА, кристалл анализатор $L_{i}$ F 200).

Как дополнительное оборудование использовали ультразвуковую ванну, модель PS-20, мощность 120 Вт, частота 40 к Гц и лабораторные весы OHAUS PA 64 (65/0,0001 г). Как неионный ПАВ применяли тритон $\mathrm{X}$ -

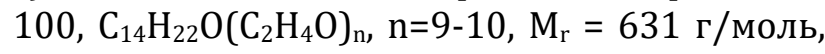
$\mathrm{KKM}=2,9 \cdot 10^{-4}$ моль/л. Для приготовления растворов стандартов металлов использовали ацетилацетон, ацетилацетонаты меди, цинка, свинца, кадмия димедонат ртути (I). Исходная концентрация растворов металлов для приготовления градуировочных растворов равнялась 0.1 г/л. В экспериментах использовали дистиллированную воду и химические реактивы квалификации не ниже ч.д.а.

Пробоподготовка. Пробу воды, отобранную
из реки концентрированной азотной кислотой до $\mathrm{pH}$ 1.5-2.0. Далее аликвоту $250 \mathrm{~cm}^{3}$ воды упаривали до влажного остатка, добавляли 10 $\mathrm{cm}^{3} \quad 1,5 \%$ азотной кислоты и тщательно перемешивали. Образцы донных отложений высушивали на воздухе до постоянной массы.

Металлы в объектах окружающей среды в большинстве случаев находятся в виде комплексов с органическими лигандами. Поэтому состав неорганических стандартных образцов состава существенно отличается от состава растворов, которые анализируют. Это отличие существенно влияет на правильность результатов определения металлов. Поэтому для того, чтобы сделать близким состав анализируемых растворов и стандартных образцов состава, использовали достаточно прочные комплексные соединения ионов металлов с органическими лигандами [4; 7; 9]. Для интенсификации пробоподготовки использовали ультразвук $[4 ; 8 ; 9 ; 11 ; 12]$. Время обработки ультразвуком составило 20 минут. Неионное поверхностно-активное вещество Тритон $\mathrm{X}-100$ применяли для уменьшения поверхностного натяжения и увеличения дисперсности аэрозоля, что способствовало вместе с переводом ионов металлов в комплексы с ацетилацетоном большей полноте атомизации [9; 13]. Для образования ацетилацетонатов металлов к анализируемым растворам добавляли ацетилацетон. Градуировочные растворы готовили из стандартных образцов состава водных растворов ионов металлов (Одесса, Физико-химический институт им. А.В. Богатского), а также из ацетилацетонатов металлов.

Чувствительность определяли как

$$
\operatorname{tg} \alpha=\frac{\Delta A}{\Delta C}
$$

Повышение чувствительности

рассчитывали как

$$
\Delta \mathrm{S}=\frac{\operatorname{tg} \alpha_{1}}{\operatorname{tg} \alpha_{2}}
$$

где $\operatorname{tg} \alpha_{1}$ - тангенс угла наклона градуировочной зависимости при использовании ацетилацетонатов металлов; $\operatorname{tg} \alpha_{2}$ - при использовании водных растворов ионов металлов.

\section{Результаты и их обсуждение}

В таблице 1 приведены результаты определения содержания меди, кадмия и цинка в воде р. Тигр атомно - абсорбционным методом. Оценку мешающего влияния веществ, присутствующих в р. Тигр, а также проверку правильности результатов измерений проводили методом «введено найдено». The results of determination of copper, cadmium, zinc and lead content in Tigris river water by atomic absorption spectroscopy $(n=5, P=0.95)$

Таблица 1.

Результаты определения содержания меди, кадмия, цинка и свинца в воде реки Тигр методом атомно-

\begin{tabular}{|c|c|c|c|c|c|}
\hline Element & $\bar{C} \pm \frac{t_{P, f} S}{\sqrt{n}}, \mathrm{mg} / \mathrm{L}$ & $\mathrm{S}_{\mathrm{r}}$ & Added, mg/L & $\begin{array}{l}\text { Found, mg/L } \\
\bar{C} \pm \frac{t_{P, f} S}{\sqrt{n}}\end{array}$ & $\mathrm{~S}_{\mathrm{r}}$ \\
\hline $\mathrm{Cu}$ & $0.06 \pm 0.01$ & 0.01 & 0.10 & $0.16 \pm 0.01$ & 0.01 \\
\hline $\mathrm{Cd}$ & $0.012 \pm 0.002$ & 0.02 & 0.05 & $0.06 \pm 0.02$ & 0.02 \\
\hline $\mathrm{Zn}$ & $0.24 \pm 0.01$ & 0.01 & 0.30 & $0.54 \pm 0.01$ & 0.01 \\
\hline $\mathrm{Pb}$ & $0.013 \pm 0.003$ & 0.02 & 0.02 & $0.03 \pm 0.02$ & 0.02 \\
\hline
\end{tabular}
абсорбционной спектроскопии ( $\mathrm{n}=5 ; \mathrm{P}=\mathbf{0 . 9 5})$ 
Journal of Chemistry and Technologies, 2021, 29(2), 173-178

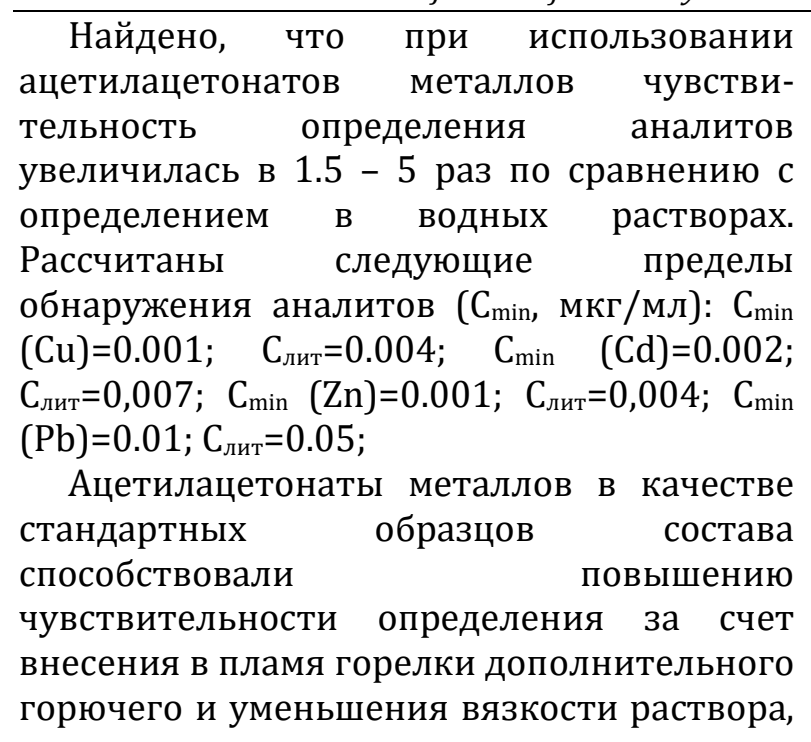

что способствовало увеличению скорости введения аналита в пламя горелки спектрометра. При этом для повышения прецизионности и точности результатов анализа в анализируемые растворы также вводили ацетилацетон [9].

C целью проверки правильности предложенной модифицированной методики атомно-абсорбционного определения содержание меди, кадмия, цинка и свинца в речной воде реки Тигр определяли также методом атомноэмиссионной спектроскопии с индуктивносвязанной плазмой (табл. 2).

Table 2

The results of determination of copper, cadmium, zinc and lead content by ICP - AES method in water of Tigris river $(n=5, P=0.95)$

Таблица 2.

Результаты определения содержания меди, кадмия, цинка и свинца методом АЭС - ИСП в воде реки Тигр (n = 5; P=0.95).

\begin{tabular}{ccccc}
\hline Element & $\bar{C} \pm \frac{t_{P, f} S}{\sqrt{n}}, \mathrm{mg} / \mathrm{L}$ & $\mathrm{S}_{\mathrm{r}}$ & $\mathrm{F}$ & $\mathrm{F}_{1,2^{* *}}$ \\
\hline $\mathrm{Cu}$ & $0.07 \pm 0.02$ & 0.01 & 3.02 & 0.84 \\
\hline $\mathrm{Cd}$ & $0.010 \pm 0.001$ & 0.02 & 2.46 & 1.01 \\
\hline $\mathrm{Zn}$ & $0.25 \pm 0.01$ & 0.01 & 1.95 & 1.84 \\
\hline $\mathrm{Pb}$ & $0.013 \pm 0.001$ & 0.02 & 2.57 & 0.96 \\
\hline
\end{tabular}

$$
\begin{aligned}
& { }^{*} F_{\text {table }}^{P=0.95, f_{1}=4, f_{2}=4}=6.39 \\
& { }^{* *} t_{\text {table }}^{P=0.95, f=10-2=8}=2.31
\end{aligned}
$$

Сравнение результатов, полученных двумя методами, проведено по F-и tкритериям (табл. 2). Как видно из табл. 2, стандартные квадратичные отклонения S обоих методов для всех металлов отличаются статистически незначимо с надежностью $95 \%$, поскольку экспериментальные значения F-критерия были во всех случаях меньше табличного значения. Также статистически незначимо по t-критерию расхождение средних арифметических результатов анализа, полученных двумя методами.

Определение содержания ртути в донных отложениях р. Тигр проведено рентгенофлуоресцентным методом с использованием стандартных образцов состава на основе димедоната ртути (I) $\left(\mathrm{Hg}_{2} \mathrm{Dm}_{2}\right)$ и сулемы $\mathrm{HgCl}_{2}$. Те же пробы донных отложений анализировали атомноабсорбционным методом холодного пара (табл. 3)

Table 3

The results of the determination of mercury in the sediments of Tigris river by $X$-ray fluorescence and atomic absorption methods $(n=5, P=0.95)$

Таблица 3 Результаты определения ртути в данных отложениях р. Тигр рентгенофлуоресцентным и атомноабсорбционным методами ( $\mathrm{n}=5 ; \mathrm{P}=0.95)$.

\begin{tabular}{lcccc}
\hline \multirow{2}{*}{ Sample } & \multicolumn{2}{c}{ X-ray fluorescence } & \multicolumn{2}{c}{ AAS } \\
\cline { 2 - 5 } & $\bar{C} \pm \frac{t_{P_{f} f} S}{\sqrt{n}}, \mathrm{mg} / \mathrm{kg}$ & $\mathrm{S}_{\mathrm{r}}$ & $\bar{C} \pm \frac{t_{P_{f}} S}{\sqrt{n}}, \mathrm{mg} / \mathrm{kg}$ & $\mathrm{S}_{\mathrm{r}}$ \\
\hline Sediment 1 & $0.40 \pm 0.03$ & 0.03 & $0.37 \pm 0.05$ & 0.05 \\
\hline Sediment 2 & $0.31 \pm 0.04$ & 0.04 & $0.29 \pm 0.07$ & 0.06 \\
\hline
\end{tabular}

Как следует из табл. 3, результаты определения ртути в донных отложениях двумя методами хорошо совпадают друг с другом, доверительные интервалы средних арифметических
Содержание ртути в донных отложениях реки Тигр примерно в полтора-два раза превышало нормативные показатели для Ирака (ПДК 0.2 мг/кг). 
Journal of Chemistry and Technologies, 2021, 29(2), 173-178

При определении ртути в донных отложениях в качестве градуировочных образцов использовали $\mathrm{Hg}_{2} \mathrm{Dm}_{2}$ и $\mathrm{HgCl}_{2}$. При использовании $\mathrm{Hg}_{2} \mathrm{Dm}_{2}$ аналитический сигнал стабилен, а при использовании $\mathrm{HgCl}_{2}$ линейно снижается с временем облучения (исследования проводили на протяжении 6 ч.) Воспроизводимость аналитических сигналов при использовании различных образцов сравнения отличается незначительно. Изучена стабильность образцов. Состав градуировочных растворов на основе $\mathrm{Hg}_{2} \mathrm{Dm}_{2}$ не меняется на протяжении как минимум полугода. В то же время образцы на основе $\mathrm{HgCl}_{2}$ стабильны не более 2 месяцев так как $\mathrm{HgCl}_{2}$ разлагается на $\mathrm{Hg}$ и $\mathrm{Cl}_{2}$ при длительном облучении. Из-за этого при использовании сулемы результаты могут содержать систематическую погрешность. Путем варьирования объема или массы образца показано, что методика не содержит значимой систематической погрешности.

\section{Выводы}

Методами спектроскопии атомно-абсорбционной спектроскопии плазмой проведено определение содержания меди, кадмия, цинка и свинца в воде реки Тигр. Методом «введено найдено» показано отсутствие влияния веществ, содержащихся в реке на правильность и воспроизводимость определения металлов. Показано, что результаты обоих методов являются равноточными, расхождение между ними незначимо и оправдано случайным разбросом. Использование неионного поверхностно-активного вещества позволило снизить пределы обнаружения металлов в 1,5-5 раз по сравнению со стандартным подходом. Кроме того, использование водных растворов Тритон Х100 повышает стабильность и гомогенность полученных растворов, уменьшает время анализа.

Рентгенофлуоресцентным методом и атомно-абсорбционным методом холодного пара определено содержание ртути в донных отложения. Найденная концентрация ртути в донных отложениях реки Тигр превышала в среднем в 1.5-2.0 раза нормативные показатели, установленные для нее в Ираке.
Новые стандартные образцы состава на основании ацетилацетонатов меди, цинка, кадмия, свинца и димедоната ртути (I), $\beta$ дикетонатов металлов приближают химический состав анализируемых образцов к образцам сравнения, это позволяет повысить прецизионность и точность измерений.

\section{Библиографические ссылки}

[1] Sim S.F. Assessment of heavy metals in water, sediment, and fishes of a large tropical hydroelectric dam in Sarawak, Malaysia / S. F. Sim, T. Y. Ling, L. Nyanti, [et al.] // Journal of Chemistry. - 2009. - Vol. 5, № 2. - P. 2-10.

[2] Förstner U. Metal pollution in the aquatic environment / U. Förstner, G. T. W. Wittmann. Berlin: Springer, 1984. - 486 p.

[3] Antolova T. Spectrophotometric determination of mercury using vortex-assisted liquid-liquid microextraction / T. Antolova, S. Zaruba, J. Šandrejová [et al.] // Turkish J. Chem. - 2016. - Vol. 40. - P. 965-973.

[4] Yurchenko O. I. Analytical signal amplification technologies in sonoluminescence spectroscopy by double-frequency ultrasound / O. I. Yurchenko, T. V. Chernozhuk, A. N. Baklanov [et al.] // Methods Objects Chem. Anal. - 2018. - Vol. 13, № 3. - P. 103109.

[5] Kazantzi V. Evaluation of polypropylene and polyethylene as sorbent packing materials in on-line preconcentration columns for trace $\mathrm{Pb}(\mathrm{II})$ and $\mathrm{Cd}(\mathrm{II})$ determination by FAAS / V. Kazantzi, E. Drosaki, A. Skok [et al.] // Microchem J. - 2019. - Vol. 148. - P. 514-520.

[6] Сухарев С.Н. Сорбционно-атомно-абсорбционное определение тяжелых металлов в природных водах / С.Н. Сухарев // Химия и технология воды. - 2012. - Т. 34, № 4. - С. 320-328.

[7] Improving food safety - increase of expressive analysis to toxic elements / I. V. Goloperov, E. A. Belova, L. V. Baklanova, A. N. Baklanov // ISJ Theoretical \& Applied Science. - 2018. - Vol. 57, № 1. - P. 260-265.

[8] Baklanov A. N. Use of ultrasound in sample preparation for the determination of mercury species by cold-vapor atomic absorption spectrometry / A. N. Baklanov, F. A. Chmilenko // J. Anal. Chem. - 2001. - Vol. 56, № 7. - P. 641-646.

[9] Юрченко О. I. Двохчастотний ультразвук в підготовці проб природних розсолів для визначення меркурію абсорбцією «холодної пари» / О. І. Юрченко, Л. В. Бакланова, Т. В. Черножук, О. М. Бакланов // Вісник Харк. нац. унту. Серія Хімія. - 2018. - Вип. 30 (53). - С. 58-66.

[10] Юрченко О. І. Моніторинг вмісту натрію, калію, кальцію та магнію в продуктах переробки молочної сироватки / О. І. Юрченко, С. М. Губський, Т. В. Черножук [та ін.] // J. Chem. Technologies. - 2020. - T. 28, № 1. - C. 27-33.

[11] Chmilenko F. A. Atomic absorption determination of metals in soils using ultrasonic sample preparation / F. A. Chmilenko, N. M. Smityuk, A. N. Balkanov // J. Anal. Chem. - 2002. - Vol. 57, № 4. - P. 313-318.

[12] Yurchenko O. I. Sonoluminescence spectroscopy as a promising new analytical method / O. I. Yurchenko, 
O. S. Kalinenko, A. N. Baklanov [et al.] // J. Appl. Spectrosc. - 2016. - Vol. 83, № 1. - P. 784-787.

[13] Vishnikin A. B. Novel indirect spectrophotometric methods for determination of phosphate and arsenate using polyoxometalates and micellar medium / A. B. Vishnikin // J. Molec. Liquids. 2005. - Vol. 118, № 1-3. - P. 51-55.

[14] Rodriguez J. C. Direct and combined methods for the determination of chromium, copper, and nickel in honey by electrothermal atomic absorption spectroscopy / J. C. Rodriguez, J. B. Garcia, C. H. Latorre [et al.] //. J. Agric. Food. Chem. - 2005. - Vol. 53, № 17. - P. 6616-6623.

[15] O`Neil G. D. Direct identification and analysis of heavy metals in solution $\mathrm{Hg}, \mathrm{Cu}, \mathrm{Pb}, \mathrm{Zn}, \mathrm{Ni})$ using in-situ electrochemical X-ray fluorescence / G. D. O`Neil, M. E. Newton, J. V. Macpherson // Anal. Chem. - 2015. - Vol. 87, № 9. - P. 4933-4940.

[16] Hutton L. A. Electrochemical X-ray fluorescence spectroscopy for trace heavy metal analysis: enhancing X-ray fluorescence detection capabilities by four orders of magnitude / L. A. Hutton, G. D. O'Neil, T. L. Read [et al.] // Anal. Chem. - 2014. Vol. 86, № 9. - P. 4566-4572.

[17] Smith M. G. Before the injection - modern methods of sample preparation for separation techniques / M. G. Smith // J. Chromatography A. - 2000. - Vol. 1000. - P. 3-27.

[18] Becker J. S. Trace and ultratrace analysis in liquids by atomic spectrometry / J. S. Becker // TrAC Trends Anal. Chem. - 2005. - Vol. 24, № 3. - P. 243254

\section{References}

[1] Sim, S. F., Ling, T. Y., Nyanti, L., Gerunsin, N., Wong, Y. E., Kho, L. P. (2009). Assessment of heavy metals in water, sediment, and fishes of a large tropical hydroelectric dam in Sarawak, Malaysia. Journal of Chemistry. $\quad 5, \quad$ (2), 2-10. https://doi.org/10.1155/2016/8923183

[2] Förstner, U., Wittmann, G. T. W. (1984). Metal Pollution in the Aquatic Environment. Berlin: Springer.

[3] Antolova, T., Zaruba, S., Šandrejová, J., Kocúrová, L., Vishnikin, A. B., Bazel', Ya., Andruch, V., Balogh, I.S. (2016). Spectrophotometric determination of mercury using vortex-assisted liquid-liquid microextraction. Turkish J. Chem. 40, 965-973. https://doi.org/10.3906/kim-1605-63

[4] Yurchenko, O. I., Chernozhuk, T. V., Baklanov, A. N., Baklanova, L. V., Kravchenko, O. A. (2018). Analytical signal amplification technologies in sonoluminescence spectroscopy by doublefrequency ultrasound. Methods Objects Chem. Anal., 13,(3), 103-109.

https://doi.org/10.17721/moca.2018.103-109

[5] Kazantzi, V., Drosaki, E., Skok, A., Vishnikin, A. B., Anthemidis, A. (2019). Evaluation of polypropylene and polyethylene as sorbent packing materials in on-line preconcentration columns for trace $\mathrm{Pb}(\mathrm{II})$ and $\mathrm{Cd}(\mathrm{II})$ determination by FAAS. Microchem J., 148, 514-520.

https://doi.org/10.1016/j.microc.2019.05.033

[6] Sukharev, S. N. (2012). Determination of heavy metals in natural water by the sorption-atomicabsorption method. J. Water Chem. Technol., 34 (4), 190-194 (in Russian). https://doi.org/10.3103/S1063455X12040042
[7] Goloperov, I. V., Belova, E. A., Baklanova, L. V., Baklanov, A. N. (2018). Improving food safety increase of expressive analysis to toxic elements. ISJ Theoretical \& Applied Science, 57(1), 260-265. https://doi.org/10.15863/tas.2018.01.57.42

[8] Baklanov, A. N., Chmilenko, F. A. (2001). Use of ultrasound in sample preparation for the determination of mercury species by cold-vapor atomic absorption spectrometry. J. Anal. Chem. 56(7), 641-646. https://doi.org/10.1023/A:1016792205748

[9] Yurchenko, O. I., Baklanova, L. V., Chernozhuk, T. V., Baklanov, O. M. (2018). Two frequency ultrasound in preparation of the samples of natural brines to determine mercury by "cold vapor" absorption. Kharkiv University. Chemical Series. 30(53), 58-66 (in Ukrainian). https://doi.org/10.26565/2220637X-2018-30-06

[10] Yurchenko, O. I., Gubskii, S. M., Chernozhuk, T. V., Baklanov, A. N., Kravchenko, O. A. (2020). Monitoring of content of sodium, potassium, calcium and magnesium in whey processed products (in Ukrainian). J. Chem. Technologies, 28(1), 27-33. https://doi.org/10.15421/082004

[11] Chmilenko, F. A., Smityuk, N. M., Balkanov, A. N. (2002). Atomic absorption determination of metals in soils using ultrasonic sample preparation. J. Anal. Chem. 57(4), 313-318. https://doi.org/10.1023/A:1014946213773

[12] Yurchenko, O. I., Kalinenko, O. S., Baklanov, A. N., Belov, E. A., Balkanova, L. V. (2016). Sonoluminescence spectroscopy as a promising new analytical method. J. Appl. Spectrosc. 83(1), 784-787. https://doi.org/10.1007/s10812-016-0250-0

[13] Vishnikin, A. B. (2005) Novel indirect spectrophotometric methods for determination of phosphate and arsenate using polyoxometalates and micellar medium. J. Molec. Liquids, 118(1-3), 51-55. https://doi.org/10.1016/j.molliq.2004.07.012

[14] Rodriguez, J. C., Garcia, J. B., Latorre, C. H., Martin, S. G., Crecente, M. P. (2005). Direct and combined methods for the determination of chromium, copper, and nickel in honey by electrothermal atomic absorption spectroscopy. J. Agric. Food. Chem., 53(17), 6616-6623. https://doi.org/10.1021/if0508870

[15] O`Neil, G. D., Newton, M. E., Macpherson, J. V. (2015). Direct identification and analysis of heavy metals in solution ( $\mathrm{Hg}, \mathrm{Cu}, \mathrm{Pb}, \mathrm{Zn}, \mathrm{Ni})$ using in-situ electrochemical X-ray fluorescence. Anal. Chem., 87(9), 4933-4940. https://doi.org/10.1021/acs.analchem.5b00597

[16] Hutton, L. A., O'Neil, G. D., Read, T. L., Ayres, Z. J., Newton, M. E., Macpherson, J. V. (2014). Electrochemical X-ray fluorescence spectroscopy for trace heavy metal analysis: enhancing X-ray fluorescence detection capabilities by four orders of magnitude. Anal. Chem., 86(9), 4566-4572. https://doi.org/10.1021/ac500608d

[17] Smith, M. G. (2000). Before the injection - modern methods of sample preparation for separation techniques. J. Chromatography A, 1000, 3-27. https://doi.org/10.1016/S0021-9673(03)00511-9

[18] Becker, J. S. (2005). Trace and ultratrace analysis in liquids by atomic spectrometry. TrAC Trends Anal. Chem. 24(3), 243-254. https://doi.org/10.1016/j.trac.2004.12.003 